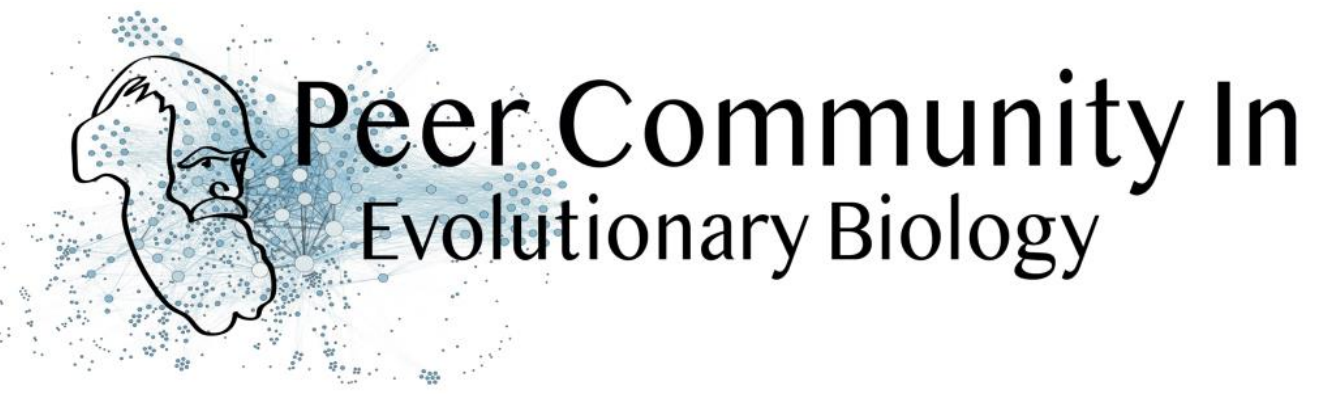

\title{
The ecology of evolutionary transitions to multicellularity
}

Dustin Brisson based on reviews by 2 anonymous reviewers

A recommendation of:

Caroline J Rose, Katrin Hammerschmidt, Yuriy Pichugin and Paul B Rainey. Meta-population structure and the evolutionary

Open Access transition to multicellularity (2020), bioRxiv, 407163, ver. 5 peerreviewed by Peer Community in Evolutionary Biology.

\section{$10.1101 / 407163$}

Published: 4 May 2019

Copyright: This work is licensed under the Creative Commons

Attribution-NoDerivatives 4.0

International License. To view a

copy of this license, visit

http://creativecommons.org/licen ses/by-nd/4.0/
Submitted: 04 April 2019, Recommended: 03 May 2020 Cite this recommendation as:

Dustin Brisson (2020) The ecology of evolutionary transitions to multicellularity. Peer Community in Evolutionary Biology, 100099. 10.24072/pci.evolbiol.100099

The evolutionary transition to multicellular life from free-living, single-celled ancestors has occurred independently in multiple lineages [1-5]. This evolutionary transition to cooperative group living can be difficult to explain given the fitness advantages enjoyed by the non-cooperative, single-celled organisms that still numerically dominate life on earth $[1,6,7]$. Although several hypotheses have been proposed to explain the transition to multicellularity, a common theme is the abatement of the efficacy of natural selection among the single cells during the free-living stage and the promotion of the efficacy of selection among groups of cells during the cooperative stage, an argument reminiscent of those from George Williams' seminal book $[8,9]$. The evolution of 
life cycles appears to be a key step in the transition to multicellularity as it can align fitness advantages of the single-celled 'reproductive' stage with that of the cooperative 'organismal' stage [9-12]. That is, the evolution of life cycles allows natural selection to operate over timescales longer than that of the doubling time of the free-living cells [13]. Despite the importance of this issue, identifying the range of ecological conditions that reduce the importance of natural selection at the single-celled, free-living stage and increase the importance of selection among groups of cooperating cells has not been addressed empirically. Rose et al [14] addressed this issue in a series of real time evolution experiments with bacteria in which they varied the intensity of between-group versus individuallevel selection. Central to the experiment is an ecological scaffold that requires lineages to switch between free-living (reproductive) and group-living (organismal) life-stages. One ecological scenario severely limited natural selection at the single-celled, free-living stage by maintaining separation among the reproductive propagules originating from different organisms (groups of cells derived from a single ancestral cell). A second ecological scenario mixed the reproductive propagules from different organisms, leading to severe competition between single cells derived from both the same and other 'organisms'. These ecological scenarios lead to very different evolutionary outcomes. Limiting competition, and thus natural selection, at the reproductive propagule stage promoted traits that favored organismal fitness at the expense of cell division, while competition among single-cells favored traits that promote cell-level traits at the expense of group-level traits. The authors investigate a range of measures of cell and group-level performance in order to understand the mechanisms favoring organismal versus single-cell fitness. Importantly, an evolutionary tradeoff between traits promoting organismal fitness and single-cell fitness appears to constrain maximizing fitness of both phases, especially when strong natural selection acts on the single-cell stage. This article is incredibly thorough and utilizes multiple experiments and levels of argument in order to support the conclusions. The authors include considerable discussion of broader topics surrounding the immediate hypotheses throughout the article, which add both clarity and complexity. The complexity of the experiments, results, and the topic 
itself lead to a thought-heavy article in a throwback to the monographs of old; expect to read each section multiple times.

\section{References}

[1] Maynard Smith, J. and Szathmáry, E. (1995). The Major Transitions in Evolution. Oxford, UK: Freeman. p 346. [2] Bonner, J. T. (1998). The origins of multicellularity. Integrative Biology: Issues, News, and Reviews: Published in Association with The Society for Integrative and Comparative Biology, 1(1), 27-36. doi: 10.1002/(SICI)1520-6602(1998)1:1<27::AID-INBI4>3.0.CO;2-6 [3] Kaiser, D. (2001). Building a multicellular organism. Annual review of genetics, 35(1), 103123. doi: 10.1146/annurev.genet.35.102401.090145 [4] Medina, M., Collins, A. G., Taylor, J. W., Valentine, J. W., Lipps, J. H., Amaral-Zettler, L., and Sogin, M. L. (2003). Phylogeny of Opisthokonta and the evolution of multicellularity and complexity in Fungi and Metazoa. International Journal of Astrobiology, 2(3), 203 211. doi: 10.1017/S1473550403001551 [5] King, N. (2004). The unicellular ancestry of animal development. Developmental cell, 7(3), 313-325. doi: 10.1016/j.devcel.2004.08.010 [6] Michod R. E. (1999). Darwinian Dynamics. Evolutionary Transitions in Fitness and Individuality. Princeton, NJ: Princeton Univ. Press. p 262. [7] Lynch, M. (2007). The frailty of adaptive hypotheses for the origins of organismal complexity. Proceedings of the National Academy of Sciences, 104(suppl 1), 8597-8604. doi: 10.1073/pnas.0702207104 [8] Williams, G. C. (1996). Adaptation and Natural Selection, Reprint edition. Princeton, NJ: Princeton Univ. Press. [9] Grosberg, R. K., and Strathmann, R. R. (2007). The evolution of multicellularity: a minor major transition?. Annu. Rev. Ecol. Evol. Syst., 38, 621-654. doi: 10.1146/annurev.ecolsys.36.102403.114735 [10] Buss, L. W. (1987). The Evolution of Individuality. Princeton, NJ: Princeton Univ. Press. [11] Godfrey-Smith, P. (2009). Darwinian Populations and Natural Selection. Oxford University Press, USA. [12] Van Gestel, J., and Tarnita, C. E. (2017). On the origin of biological construction, with a focus on multicellularity. Proceedings of the National Academy of Sciences, 114(42), 11018-11026. doi: 10.1073/pnas.1704631114 [13] Black, A. J., Bourrat, P., and Rainey, P. B. (2020). Ecological scaffolding and the evolution of individuality. Nature Ecology \& Evolution, 4(3), 426-436. doi: 10.1038/s41559-019-1086-9 [14] Rose, C. J., 
Hammerschmidt, K., Pichugin, Y. and Rainey, P. B. (2020). Meta-population structure and the evolutionary transition to multicellularity. bioRxiv, 407163, ver. 5 peer-reviewed and recommended by $\mathrm{PCl}$ Evolutionary Biology. doi: $10.1101 / 407163$

\section{Revision round \#3}

2020-04-29

Comments from the managing board:

Dear Paul,

Dustin Brisson is satisfied with your response and is willing to recommend your preprint. He made some minor edits and suggestions. You'll find them in the recommender's annotations PDF version of your preprint (if you don't find this file, let us know by return mail).

Could you also make the following changes before posting a final version (it would be version 5) on bioRxiv.

\section{Mandatory modifications.}

In order to reach a better referencing and greater visibility of your recommended preprint, we suggest you to do the following modifications : -add the following sentence in the acknowledgements: "Version 5 of this preprint has been peerreviewed and recommended by Peer Community In Evolutionary Biology (https://doi.org/10.24072/pci.evolbiol.100099)"

$\Rightarrow$ If you use bioRxiv to post your preprint, add this sentence also in a footnote of the 1st page of your pdf, it will be interpreted as a specific footnote section by bioRxiv. Note that this DOI is not the DOI of your article, but the DOI of the recommendation text. The DOI of your article remains unchanged. Doing so is very important because it would: -indicate to readers that, unlike many other preprint in this server, your pre-print has been peer-reviewed and recommended. 


\section{Biol}

-make visible this information in Google Scholar search (which is quite important).

(ii) In addition, we suggest you to remove line numbering from the preprint.

-We need a picture/photo for which you own the rights that could serve as a thumbnail/illustration for your article on the web site of $\mathrm{PCl}$. It can be a figure of the article.

Optional modifications. $=>$ We advise you to use templates (word docx template and a latex template) to format your preprint in a $\mathrm{PCl}$ style. This is optional. Here is the links of the templates: https://peercommunityin.org/templates/

For word template, please be careful to correctly update all text in these templates (doi, authors' names, address, title, date, recommender first name and family name ...). Please be careful to choose the badges "Open Code" and "Open Data" only if appropriate (in addition to the "Open Access" and "Open PeerReview" badges). If some of the reviewers are anonymous, indicate for example "Albert Ayler and two anonymous reviewer". Indicate in the "cite as" box the right version of your preprint. It is version 5 .

For Latex template, main.tex and sample.bib should be filled. Please be careful to choose the badges "Open Code" and "Open Data" only if appropriate (in addition to the "Open Access" and "Open Peer-Review" badges). Preambule_xxx.tex should be modified (comment lines 115, 117) to select badges. If some of the reviewers are anonymous, indicate for example "Albert Ayler and two anonymous reviewer". In sample.bib, indicate the right version of your preprint. It is version 5 .

I hope this is clear. Do not hesitate to ask any help if you need. Once you have made these modifications (plus those requested by the recommender), you should upload a new version of the article on the preprint server. Please tell us when you have done so. Thanks in advance All the best

Preprint DOI: 10.1101/407163

\section{Author's reply:}

Dear Dustin 
Thank you again for the careful attention. I have incorporated a number of minor alterations in response, including a modified Fig $2 b$ (with $y$-axis scale redrawn) and have picked up a few additional details. I've also altered the format so that it fits with that suggested by PCI EB. I have uploaded the final version to the bioRxiv.

I believe all is now done. Again, I very much appreciate the time and effort that you and the reviewers gave to this paper. It is definitely the better for it.

Paul

\section{Revision round \#2}

\section{0-04-07}

This is a much more clear manuscript of a very interesting and important piece of work. The major theoretical concern, that of the impact of relatedness and inclusive fitness, has been addressed. The writing is also much more clear, although the manuscript is still more confusing than necessary. I would like to encourage the authors to invest some time doing some minor reorganization and address several areas that are written less than perfectly clear. I should note that much of the confusion does not impact the overall conclusions. However, I found myself stuck in multiple paragraphs trying to figure out exactly how the Mixed ecology strains were selected, when traits were measured, how fitness was calculated. I think I did figure it out (mostly), but I also think there is no obvious reason to have readers struggle at these points. My suggestion (just a suggestion) that I think would have helped me would be 1 . Put the methods after the intro.

1a. Making the methods more recipe-like; that is, exactly how were the experiments done. 1b. Organize as Maturation vs dispersal. Within these, describe exactly what was done in the Non-Mix; then describe completely what was done in the Mix (repeating is ok if it helps clarity) 2. Results as results only (no or very little discussion) 3. Discussion integrated with conclusions Again, this is just a suggestion, but I think it would help the clarity for most readers. 
I am including a mark-up of the pdf as I read it (in Adobe, let me know if you cannot see the comments). Some of the comments are broad ideas I had while reading it that are not suggestions to change anything, others are comments that warrant some attention. I will leave it to your judgement which belong to the former and which to the latter category.

Dustin Brisson

Additional requirements of the managing board: As indicated in the 'How does it work?' section and in the code of conduct, please make sure that: -Data are available to readers, either in the text or through an open data repository such as Zenodo (free), Dryad (to pay) or some other institutional repository. Data must be reusable, thus metadata or accompanying text must carefully describe the data.

-Details on quantitative analyses (e.g., data treatment and statistical scripts in R, bioinformatic pipeline scripts, etc.) and details concerning simulations (scripts, codes) are available to readers in the text, as appendices, or through an open data repository, such as Zenodo, Dryad or some other institutional repository. The scripts or codes must be carefully described so that they can be reused. -Details on experimental procedures are available to readers in the text or as appendices.

-Authors have no financial conflict of interest relating to the article. The article must contain a Conflict of interest disclosure paragraph before the reference section containing this sentence: The authors of this preprint declare that they have no financial conflict of interest with the content of this article. If appropriate, this disclosure may be completed by a sentence indicating that some of the authors are $\mathrm{PCI}$ recommenders: $\mathrm{XXX}$ is one of the $\mathrm{PCI} X X X$ recommenders.

Preprint DOI: 10.1101/407163

Reviewed by anonymous reviewer, 2020-03-24 03:05

The setups of the experiments and methods used are better explained now. I appreciate the authors' time and efforts into revising and reorganizing the manuscript. Also, most of my previous concerns were addressed, I just want to make sure I understand how group-level fitness of evolved lines was measured. Based on line 194-196 this was done through competition with a marked SM reference strain "over the timescale of one generation of the mat life cycle". Does 
this generation scale refer to Figure 1a? Was the experiment conducted in a meta-population structure with eight competing groups, four of them started with the ancestral SM strain and four of them started with the evolved clones? Were propagules mixed during the dispersal phase?

Another minor comment, on line 178-180, "one colony of the most dominant WS type ... was transferred to a fresh microcosm to begin the Maturation Phase of the next 'mat' generation". How was the most dominant WS type determined?

\section{Author's reply:}

\section{Download author's reply (PDF file)}

\section{Revision round \#1}

\section{9-06-02}

Dear Paul. First let me apologize for the delay. It took some time as the semester was winding down to find appropriate reviewers with whom you are not currently collaborating. The three reviewers ( 2 others and myself) had similar takes on the manuscript and would like to see another version addressing the comments. Below you will find my comments and an as-I-went-along markup with thoughts which could be useful. I look forward to seeing your replies best Dustin

Dustin's review This is an interesting set of experiments that I think can have an impact if it were modified in a few ways. One of the most important is the presentation of the work. The writing is difficult to follow because it lacks some structure and is uses poetic language where more direct language would be more clear. More importantly, it is not always clear what has been done and what it means. A general re-write that makes the descriptions more concise and direct would dramatically improve the audience breadth that could be impacted by this article.

My understanding of this work is that the experimental setup forced nearcomplete group selection in one scenario and ONLY individual selection (selection between groups cannot operate if only the most successful individual within any 


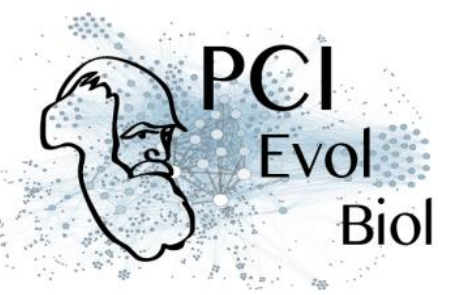

group seeds the next generation) in the other. What was less clear was the major conclusion. Some parts of the writing (see the final paragraph) make it seem like the point is to demonstrate that group selection can have an impact on evolution, but that is well established at this point. I think the major conclusion is that group selected lines had better group fitness (although I am not entire clear how group fitness was measured). What is missing is a why; why are those with better group traits less likely to go extinct? I am pretty sure that it is bc there is no transition in the time frame, but it is never clearly described. I think this is mostly a presentation issue.

A more real issue is that of kin selection, or it's absence from this paper. Kin selection seems like the most obvious theory to explain the results. Here, individuals in groups that are kin can benefit a lot from cooperative actions whereas individuals in groups that are non-kin suffer much more than they benefit. That is, in the non-mixed lines the genome as a whole does better (gets into future generations) by cooperating bc nearly all of its genes make it through the life cycle regardless of which SM cell makes it (some bet hedging); in the mixed lines it is best to out compete the other strains as only the dominant WS strain across all mesocosms seeds the next phase whereas all of the SM across all replicates are mixed and thus have a chance in the next generation (mostly by being better WS once they mutate back). Can you present some discussion of these results in terms of kin selection and relatedness, as oppose to "structured".

As I read through the ms I make notes which I have uploaded. These are written as I go with no editing so they are likely curt sounding and may be "premature" (written before coming to another section that explains.)

Additional requirements of the managing board: As indicated in the 'How does it work?' section and in the code of conduct, please make sure that: -Data are available to readers, either in the text or through an open data repository such as Zenodo (free), Dryad (to pay) or some other institutional repository. Data must be reusable, thus metadata or accompanying text must carefully describe the data.

-Details on quantitative analyses (e.g., data treatment and statistical scripts in $\mathrm{R}$, bioinformatic pipeline scripts, etc.) and details concerning simulations (scripts, codes) are available to readers in the text, as appendices, or through an open 
data repository, such as Zenodo, Dryad or some other institutional repository. The scripts or codes must be carefully described so that they can be reused. -Details on experimental procedures are available to readers in the text or as appendices. -Authors have no financial conflict of interest relating to the article. The article must contain a "Conflict of interest disclosure" paragraph before the reference section containing this sentence: "The authors of this preprint declare that they have no financial conflict of interest with the content of this article." If appropriate, this disclosure may be completed by a sentence indicating that some of the authors are $\mathrm{PCl}$ recommenders: "XXX is one of the $\mathrm{PCI} X X X$ recommenders."

Preprint DOI: 10.1101/407163

Reviewed by anonymous reviewer, 2019-05-20 11:09

\section{Download the review (PDF file)}

Reviewed by anonymous reviewer, 2019-05-30 23:33

The manuscript by Rose et al examined the impact of population structure on nascent multicellular organisms. Two experimental treatments (Non-Mixed vs Mixed Ecology) both imposed a two-phase life cycle; nevertheless, during the propagule phase of the Mixed Ecology, single cells from different groups were mixed and competing against each other. The system used (P. fluorescens, WS and SM mutants) allows measurements for group and cell fitness, as well as various life cycle parameters, and thus provides insights into how selection acts at different levels. The results show that competition in the propagule phase resulted in higher cell fitness at the expense of group fitness, suggesting the importance of structured environment when multicellular organisms just evolved.

Main comments: 1- It is somehow difficult to follow through the text from Introduction to Results without relevant methods explained or mentioned.

Line 178-180 (also line 552-553), it is not clear to me how group fitness was measured, especially given that how it was measured is important to interpret the results. Does "ability to leave group offspring" mean the proportion of cells that became "WS" cells during 3-day period in Phase II after being plated out? 


\section{Biol}

Line 174-176, could the authors explain more on how competition assays were conducted? For example, are marked ancestral lines WS or SM cells? Were evolved lines competing against their own ancestral lines or a common ancestor?

2- Paragraph line 250-260 is a very insightful discussion on why the density of WS cells decreased in the Non-Mixed Ecology but increased in the Mixed Ecology. If I remember correctly from Hammerschmidt et al (2014), the transition between WS and SM cells in the Non-Mixed Ecology was mainly achieved through a mutSdependent switch in wspR. This genetic composition may limit the possible WS cell types that may arise and therefore lower the competition among WS cells. I was wondering that whether such a switch evolved in the Mixed Ecology. If not, there might be more mutational space to explore and evolve different WS cells especially the ones with higher growth rate.

\section{Author's reply:}

Download author's reply (PDF file) 